\title{
Discrete Geometry-From Theory to Applications: A Case Study
}

\author{
David Gu ${ }^{1}$ and Emil Saucan ${ }^{2, *}$ \\ 1 Department of Computer Science, State University of New York at Stony Brook, Stony Brook, \\ NY 11794-4400, USA; gu@cs.sunysb.edu \\ 2 Departments of Mathematics and Electrical Engineering, Technion-Israel Institute of Technology, \\ Haifa 32000, Israel \\ * Correspondence: semil@ee.technion.ac.il; Tel.: +972-4-8294724
}

Academic Editor: Hans J. Haubold

Received: 28 November 2016; Accepted: 6 December 2016; Published: 9 December 2016

Keywords: Ricci flow; Forman curvature; complex systems; dynamic networks; change detection; peer-to-peer network

Science does not necessarily evolve along the lines that are taught to us in High School history classes and in popular films, that is, from simple to complex. In fact, quite the contrary is true in many cases. One such example is that of the Copernican (heliocentric) versus Ptolemaic (heliocentric) system: Practical evidence was ever-accumulating to contradict the second one, but the cognitive framework was too established, the resistance against change too strong and, perhaps no less important, the additions and improvements made to allow it to "work" represented such an enormous investment of energy, intelligence and technical prowess, that its proponents seemingly became enamored more with the apparatus that they developed, than with the scientific truth that they were supposed to pursue (as sadly seems to be the case again and again in Science...). For details, see the marvelously encyclopedic essay "Imaginary Geographies" in [1]. It took some courage for the paradigm shift to hold, but it brought a crystalline simplicity that contrasted with conceptual and mechanical artifices of additional celestial spheres and improbable astral movements.

This well-known example is relevant to us because it makes us realize that the evolution of Differential Geometry is, by no means, unique amongst the Sciences. Indeed, due to historical reasons (which are beyond the scope of this short editorial) Differential Geometry not only developed with a presumption of high smoothness of the objects it studied, it also evolved towards a more-and-more technical manipulation of complicated differential operators and their combinations, that led not only to spectacular successes but also became "The Debauch of Indices" [2] for which this is renowned (and reviled by many). The more concise, modern notation adopted at the beginning of the second half of the 20th Century, simplified notation but, alas, at the price of making the field appear even more aloof and decisively less geometric.

In contrast, Discrete Differential Geometry is both more intuitive and (in consequence) far simpler. In fact, some notions are so elementary that they can be taught to High School students, which, sometimes they are. The discrete, polyhedral versions of Gauss' Theorema Egregium and Gauss-Bonnet theorem that have their roots in the ideas of Gauss and even Descartes, represent such instances. (One should, however, never underestimate the resisting force of conservatism, even among scientists: Even only a decade or so ago, one of us was "explained" that the defect definition of curvature on triangular meshes is much harder to comprehend and handle than computing the Christoffel symbols, even though, already then, this had become a standard tool in day-to-day Graphics and Imaging practice...)

We should first understand, however, that even the notion of "discrete" in this context is not unique, but rather tends to include all those settings where geometry arises in the context of a given 
computer driven application. While usually, in Imaging and Graphics, "discrete" refers to polygonal or polyhedral meshes, in other contexts, such as Biological or Social Networks, the term applies to graphs (networks), whereas when considering the so-called clouds of points, that appear again in Imaging, Graphics as well as in Manifold Learning, the notion is applied to proper discrete sets (the above mentioned "clouds"). However, the Computer Science motivation or use is not a sine qua non one, at least not an immediate or conscious one, as modern Mathematics is prone more-and-more to study such structures, for their own sake, independent of (or at least not mainly for) their practical uses.

A perfect illustration of both the flexibility, variability and success of the Discrete (Differential) Geometric approach is that of the various notions of discrete Ricci curvature. For this reason, we chose this subject dear to both our hearts to illustrate the power of expression, elegance and usefulness of the this field of study.

Until quite recently, Ricci curvature has been quite a specious notion in the field of Differential Geometry. However, this has changed with the emergence of G. Perelman's far reaching work on Hamilton's Ricci flow [3,4] and the Geometrization Conjecture [5,6]. While started far earlier [7], the search for the discretization of this notion has recently gained fast momentum, spurred by Perelman's groundbreaking results.

Amongst this discretization, the most attractive, in its elegance and simplicity, and certainly the most successful as far as practical applications are concerned, is the circle packing based, combinatorial Ricci curvature and flow of Chow and Luo [8]. The full potential of this approach for concrete applications in Graphics, Medical Imaging and Communication Networks, was brought to fruition by one of us-see, e.g., [9-12], and the references therein. Reference [11] also contains an extension to 3-dimensional manifolds, while the recent article [12] presents a new generalization of the basic method. In addition, a fitting discrete uniformization theorem, guaranteeing the existence of the combinatorial Ricci flow has also been proven recently [13].

Metric curvatures have also been suggested as a possible route towards the discretization of the Ricci curvature and flow for polygonal meshes [14], and combined with Stones's approach mentioned above, as a possible approach to defining a Ricci curvature of polyhedral manifolds of dimension higher than two [15].

A different approach to the discretization of Ricci curvature has its origins in the significant effort for the generalization of Ricci curvature via the mass transportation approach (motivated largely by Gromov's work - see [16]), undertaken by Lott and Villani [17] and, independently, by Sturm [18]. Unfortunately, these deep ideas do not lend themselves easily to discretizations and, with the exception of [19], no progress seems to have been made in this direction. A more direct approach, still stemming from the same ideas, was adopted by Morgan—see e.g., [20], and his students [21]. However, even this simpler, more geometric course has not been followed in applications, with the sole exception of [22].

However, another, different view of Ricci curvature, again via optimal mass transportation, proved to be eminently suited for the geometrization of discrete structures such as graphs. This approach, due to Ollivier [23,24], captures, in the discrete context, the quantization by Ricci curvature of the rate of growth of (infinitesimal) balls. It proved to be an easy, yet powerful tool for the exploration of geometric and related analytic properties of graphs and related structures (see, for instance, [25-27]). Moreover, Ollivier's Ricci curvature proved itself to represent an excellent tool for the analysis of complex networks, in their various avatars, as communication $[28,29]$, biological [30], economical [31] or transportation [22] networks.

Unfortunately, in spite of its many successes mentioned above, Ollivier's Ricci curvature has a serious drawback that resides in the computational complexity involved (due to its very definition based on the so-called "earth movers metric") in its calculation. This is especially manifest when one wishes to go beyond the combinatorial case and compute it, as practice often dictates, on weighted, quite large networks.

However, another discretization of Ricci curvature, whose computation, even when very large, with weights both on nodes and on edges, is quite simple and of extremely low complexity, 
suggests itself as a practical alternative. This new type of discrete Ricci curvature is based on the previous theoretical work of Forman [32] and its application to Imaging is both natural and quite straightforward [33]. In its extension to complex networks [34], it captures the dispersion of the geodesics quantification aspect of the classical Ricci curvature. Furthermore, it is, by its very definition, coupled with a fitting discrete Laplacian, thus allowing not only for direct applications similar to those in Imaging, such as those mentioned above, but also, like in the by now classical setting of Imaging, for denoising via the Laplacian flow [35]. We further illustrate in this very volume its versatility in an application for the change detection in peer-to-peer networks [36].

We hope that even this very specific, restricted case, of a certain type of curvature, appertaining to the field of Differential Geometry, shows the great theoretical variety and expressive power of Discrete Geometry. However, the subject of this Special Issue transcends the (far from narrow themselves) boundaries of Differential and Riemannian Geometry, and includes discretizations of notions and ideas from Integral, Convex, Hyperbolic and Projective Geometry, and even direct applications of simple yet powerful insights of classical, Euclidean Geometry. The applications themselves are not restricted to Imaging, Graphics and Complex networks, but rather encompass Manifold Learning, Pattern Recognition, Robotics and Computer Aided Design, to mention only a few such fields that constantly employ, rely upon and benefit from the multifaceted and ever-developing field of Discrete Geometry.

We sincerely hope, therefore, that the present Special Issue will attract a large number of submissions, diverse in methods and applications, that will illustrate the beauty and modeling capability of this beautiful and kaleidoscopic field.

Conflicts of Interest: The authors declare no conflict of interest.

\section{References}

1. Eco, U. Inventing the Enemy; Houghton Mifflin Harcourt: Boston, MA, USA, 2012.

2. Spivak, M. A Comprehensive Introduction to Differential Geometry 2; Publish or Perish Inc.: Berkeley, CA, USA, 1979.

3. Hamilton, R. Three-manifolds with positive Ricci curvature. J. Differ. Geom. 1982, 17, 255-306.

4. Hamilton, R. The Ricci Flow on Surfaces. AMS Contemp. Math. 1986, 71, 237-261.

5. Perelman, G. The entropy formula for the Ricci flow and its geometric applications. arXiv 2002, arxiv:math.DG/0211159.

6. Perelman, G. Ricci flow with surgery on three manifolds. arXiv 2003, arxiv:math.DG/0303109.

7. Stone, D.A. A combinatorial analogue of a theorem of Myers. Ill. J. Math. 1976, 20, 551-554.

8. Chow, B.; Luo, F. Combinatorial Ricci flows on surfaces. J. Differ. Geom. 2003, 63, 97-129.

9. Gu, X.D.; Yau, S.-T. Computational Conformal Geometry; Higher Education Press and International Press: Beijing, China, 2008.

10. Gao, G.; Gu, X.D.; Luo, F. Discrete Ricci flow for geometric routing. In Encyclopedia of Algorithms; Kao, M.-Y., Ed.; Springer: Berlin, Germany, 2016; pp. 556-563.

11. Yin, X.; Jin, M.; Luo, F.; Gu, X.D. Discrete curvature flow for hyperbolic 3-manifolds. Emerg. Trends Vis. Comput. 2008, 5416, 38-74.

12. Zeng, M.; Zhang, W.; Guo, R.; Luo, F.; Gu, X.D. Survey on discrete surface Ricci flow. J. Comput. Sci. Technol. 2016, 30, 598-613.

13. Gu, X.; Luo, F.; Sun, J.; Wu, T. A discrete uniformization theorem for polyhedral surfaces. arXiv 2016, arXiv:1309.4175.

14. Saucan, E. A metric Ricci flow for surfaces and its applications I. Geom. Imag. Comput. 2015, 1, $259-301$.

15. Gu, X.D.; Saucan, E. Metric Ricci curvature for PL manifolds. arXiv 2013, arXiv:1203.1592.

16. Gromov, M. Metric Structures for Riemannian and Non-Riemannian Spaces; In Progress in Mathematics; Birkhauser: Boston, MA, USA, 1999.

17. Lott, J.; Villani, C. Ricci curvature for metric-measure spaces via optimal transport. Ann. Math. 2009, $169,903-991$. 
18. Sturm, K.-T. On the geometry of metric measure spaces. Acta Math. 2006, 196, 65-131.

19. Bonciocat, A.-I.; Sturm, K.-T. Mass transportation and rough curvature bounds for discrete spaces. J. Funct. Anal. 2009, 256, 2944-2966.

20. Morgan, F. Manifolds with density. Not. Am. Math. Soc. 2005, 52, 853-858.

21. Corwin, I.; Hoffman, N.; Hurder, S.; Šešum, V.; Xu, Y. Differential geometry of manifolds with density. Rose Hulman Undergrad. J. Math. 2006, 7, 1-15.

22. Li, W.; Gu, J.; Liu, S.; Zhu, Y.; Deng, S.; Zhao, L.; Han, J.; Cai, X. Optimal transport in worldwide metro networks. arXiv 2014, arXiv:1403.7844.

23. Ollivier, Y. Ricci curvature of Markov chains on metric spaces. J. Funct. Anal. 2009, 256, 810-864.

24. Ollivier, Y. A survey of Ricci curvature for metric spaces and Markov chains. Probab. Approach Geom. 2010, 57, 343-381.

25. Bauer, F.; Jost, J.; Liu, S. Ollivier-Ricci curvature and the spectrum of the normalized graph Laplace operator. Math. Res. Lett. 2012, 19, 1185-1205.

26. Jost, J.; Liu, S. Ollivierís Ricci curvature, local clustering and curvature-dimension inequalities on graphs. Discret. Comput. Geom. 2014, 51, 300-322.

27. Loisel, B.; Romon, P. Ricci curvature on polyhedral surfaces via optimal transportation. Axioms 2014, 3, 119-139.

28. Ni, C.-C.; Lin, Y.-Y.; Gao, J.; Gu, X.D.; Saucan, E. Ricci curvature of the Internet topology. In Proceedings of the IEEE Conference on Computer Communications (INFOCOM), Hong Kong, China, 26 April-1 May 2015; pp. 2758-2766.

29. Wang, C.; Jonckheere, E.; Banirazi, R. Wireless Network Capacity versus Ollivier-Ricci Curvature under Heat-Diffusion (HD) Protocol. In Proceedings of the American Control Conference (ACC), Portland, OR, USA, 4-6 June 2014.

30. Sandhu, R.; Georgiou, T.; Reznik, E.; Zhu, L.; Kolesov, I.; Senbabaoglu, Y.; Tannenbaum, A. Graph curvature for differentiating cancer networks. Sci. Rep. 2015, 5, 12323.

31. Sandhu, R.; Georgiou, T.; Tannenbaum, A. Market fragility, systemic risk, and Ricci curvature. arXiv 2015, arXiv:1505.05182.

32. Forman, R. Bochner's method for cell complexes and combinatorial Ricci curvature. Discret. Comput. Geom. 2003, 29, 323-374.

33. Appleboim, E.; Saucan, E.; Zeevi, Y.Y. Ricci curvature and Flow for image denoising and superesolution. In Proceedings of 20th European Signal Processing Conference (EUSIPCO), Bucharest, Romania, 27-31 August 2012; pp. 2743-2747.

34. Sreejith, R.P.; Mohanraj, K.; Jost, J.; Saucan, E.; Samal, A. Forman curvature for complex networks. J. Stat. Mech. 2016, doi:10.1088/1742-5468/2016/06/063206.

35. Weber, M.; Saucan, E.; Jost, J. Characterizing Complex Networks with Forman-Ricci curvature and associated geometric flows. arXiv 2016, arXiv:1607.08654.

36. Weber, M.; Jost, J.; Saucan, E. Forman-Ricci flow for change detection in large dynamical data sets. arXiv 2016, arXiv:1604.06634.

(c) 2016 by the authors; licensee MDPI, Basel, Switzerland. This article is an open access article distributed under the terms and conditions of the Creative Commons Attribution (CC-BY) license (http://creativecommons.org/licenses/by/4.0/). 